\title{
CARACTERIZACIÓN DEL MODELO PEDAGógiCo DESARROLLADO POR LOS DOCENTES DE LA FACULTAD DE INSTRUMENTACIÓN QUIRÚRGICA DE LA FUCS
}

Sandra Giovanna Cortés Cárdenas*, Licet Villamizar Gómez**, Sandra Catalina Guerrero Aragón***

\section{Resumen}

Introducción: la ley 784 del 23 de diciembre de 2002 reglamentó el ejercicio de la instrumentación quirúrgica profesional, lo cual exigió que la educación en este programa asegurara una sólida formación del estudiante siguiendo los lineamientos pedagógicos establecidos en el proyecto educativo institucional de la FUCS. Objetivo: caracterizar el modelo pedagógico aplicado por los docentes de la facultad entre 2006 y 2008. Métodos: estudio observacional descriptivo de corte transversal. Se construyó un instrumento de recolección de datos y se aplicó a $\mathbf{4 2}$ docentes de la facultad, recolectando la información correspondiente a la metodología de enseñanza desarrollada durante la clase. Resultados: la metodología más frecuente es la clase magistral con apoyo audiovisual $43 \%$ y los talleres de reconocimiento basados en los temas de la asignatura $38 \%$. Conclusiones: la orientación del proceso de enseñanza aprendizaje desarrollada por los docentes de la facultad corresponde al modelo pedagógico establecido en el PEI de la institución, pero es importante revisar la metodología didáctica desarrollada durante la clase.

Palabras clave: caracterización, educación, modelo pedagógico, instrumentación quirúrgica.

Abreviaturas: IES, institución de educación superior; PEI, proyecto educativo institucional.

\section{DESCRIPTION OF THE PEDAGOGICAL MODEL DEVELOPED BY TEACHERS OF THE FUCS SURGICAL INSTRUMENT TECHNOLOGY PROGRAM}

\begin{abstract}
Introduction: Law 784 of December 232002 regulated professional surgical instrument technology practice requiring this program to guarantee a sound training of the student following the pedagogical guidelines established by the FUCS Institutional Education Program (IEP). Objective: to describe the pedagogical model used by teachers of this program between 2006 and 2008. Methods: an observational descriptive cross-sectional study was performed. A data collection instrument was designed and used to survey $\mathbf{4 2}$ teachers of the program. Information on the teaching methodology developed during a class was gathered. Results: the most frequent methodologies used are lectures with audio visual aids (43\%) and hands-on workshops based on course topics (38\%). Conclusions: the teaching-learning process developed by faculty members of the program corresponds to the pedagogical framework established by the FUCS's IEP, however, it is important to review the didactic methodology developed during a class.
\end{abstract}

Key words: description, education, pedagogical model, surgical instrument technology.

Fecha recibido: diciembre 10 de 2012 - Fecha aceptado: marzo 12 de 2013

* Profesora Asistente., Facultad de Instrumentación Quirúrgica, Fundación Universitaria de Ciencias de la Salud. Bogotá DC, Colombia.
** Profesora Asistente, División de Investigaciones, Fundación Universitaria de Ciencias de la Salud. Bogotá DC, Colombia.

**** Instructora Asociada, Facultad de Instrumentación Quirúrgica, Fundación Universitaria de Ciencias de la Salud. Bogotá DC, Colombia. 


\section{Introducción}

En Colombia existen diferentes leyes y decretos que reglamentan la oferta y desarrollo de los programas de educación superior, los cuales establecen condiciones institucionales y específicas. Esta normatividad hace parte de los procesos de calidad de las IES evaluados por el Ministerio de Educación Nacional (MEN) ${ }^{1}$. La ley 1.188 de 2008 "por la cual se regula el registro calificado de programas de educación superior y se dictan otras disposiciones" en el artículo dos determina de manera específica el uso adecuado y eficiente de los medios educativos de enseñanza que faciliten el aprendizaje y permitan que el profesor sea un orientador, y el estudiante autónomo y participante. ${ }^{2}$ La ley 115 de 1994 o general de educación ${ }^{3}$, reglamenta el PEI y establece que este documento debe definir entre otros, los principios, fines, recursos docentes disponibles en la institución, los lineamientos y el modelo pedagógico que guía el proceso de enseñanza aprendizaje propios para la formación integral del estudiante. Con el fin de promover la calidad en la educación y dar cumplimiento a lo estipulado en la ley 115 , la FUCS desarrolló en 2001 el PEI, el cual contiene los diferentes procesos y fundamentos que orientan la administración y gestión de los programas de la FUCS y estableció el aprendizaje significativo como modelo pedagógico de la institución. Este modelo señala que el aprendizaje debe estar centrado en el alumno como persona total ${ }^{4}$, haciéndolo partícipe del proceso de enseñanza aprendizaje desde la planeación, la selección de las actividades y la metodología didáctica, que le permita el descubrimiento, la comprensión del mundo exterior y la interacción con situaciones del medio real propias de cada disciplina. En 2008 se realizó una evaluación del PEI de la FUCS y la modificación de algunos de sus lineamientos, entre ellos el modelo pedagógico, estableciendo el conductismo, el constructivismo y el aprendizaje significativo como sus principales referentes pedagógicos para estructurar las funciones sustantivas de docencia, investigación y proyección social. ${ }^{5}$ Para el área de la salud existe la resolución 2.772 de noviembre de 2003 “por la cual se definen las características específicas de calidad para los programas de pregrado en ciencias de la salud" y de manera específica el artículo dos señala que los programas de formación académica profesional en ciencias de la salud, de acuerdo con su enfoque, deben ser coherentes con la fundamentación teórica y metodológica de cada campo profesional. ${ }^{6}$ Para el programa de instrumentación quirúrgica existe la ley 784 del 23 de diciembre de 2002. ${ }^{7}$ la cual reglamentó la carrera como programa profesional del área de la salud y estableció el desempeño del instrumentador quirúrgico en áreas diferentes a la asistencial. En cuanto a la enseñanza estableció que solo podrá ser permitida a las instituciones autorizadas por el gobierno nacional $^{6}$, las cuales a su vez deben cumplir la normatividad del MEN. Teniendo en cuenta el marco legal de la educación en Colombia, la reglamentación de instrumentación quirúrgica como programa profesional del área de la salud y considerando la concepción del modelo pedagógico como la representación de las relaciones que predominan durante el proceso de enseñanza aprendizaje ${ }^{8}$, el trabajo de investigación está dirigido a caracterizar el modelo pedagógico aplicado por los docentes de la facultad de instrumentación quirúrgica de la FUCS e identificar su correspondencia con el modelo pedagógico de aprendizaje significativo establecido en el PEI de la Fundación durante el desarrollo de su actividad académica entre 2006 y 2008, analizando los elementos que hacen parte del currículo y facilitan un enfoque del modelo pedagógico desarrollado como son: relación maestro alumno, metodología, recursos didácticos, metas de la educación, evaluación, construcción curricular y construcción de significados. ${ }^{9}$

\section{Métodos}

Se utilizó un diseño observacional descriptivo de corte transversal, con un total de 42 docentes del programa de instrumentación quirúrgica de la FUCS. Para la construcción del instrumento de recolección de datos se realizó una prueba piloto con siete docentes, que caracterizaron las observaciones pertinentes; después se realizó una validez de apariencia por dos expertos en salud y educación. Los datos fueron tabulados en Microsoft Excel, la calidad de los mismos fue revisada en su totalidad y para el análisis estadístico se utilizó el programa Stata10.0 ®. 


\section{Resultados}

Se analizaron 42 docentes, 20 de ellos con dedicación de tiempo completo, 21 de hora cátedra y uno de tiempo parcial, $62 \%$ de género femenino. En la Tabla 1 se presentan las características de formación académica y vinculación de los docentes de la facultad de instrumentación quirúrgica, $35 \%$ están en un rango de edad entre 20 y 30 años, $38 \%$ tienen una vinculación laboral con la FUCS entre dos y cinco años y $64 \%$ poseen formación académica de posgrado. La Tabla 2 presenta las estrategias pedagógicas y didácticas desarrolladas por los docentes en 2006 , el $42 \%$ de los docentes dictan la clase magistral con apoyo audiovisual como método más frecuente, el taller de reconocimiento de instrumental médico quirúrgico es utilizado por $38 \%$ de los docentes. La Tabla 3 muestra la concepción sobre el papel del docente en la facultad de instrumentación quirúrgica de la FUCS, el $47.6 \%$ considera que cumple como asesor en el proceso de enseñanza aprendizaje, mientras $31 \%$ asevera que es acompañante en el proceso.

La Figura 1 explica las correspondencias entre las diferentes variables. Los docentes que aplican la metodología didáctica de taller interactivo se caracterizan por tener una vinculación con la FUCS mayor a once años y consideran que la responsabilidad de la construcción de los significadoses compartida entre el docente y el estudiante, involucrándose los dos actores dentro del proceso.

La metodología didáctica de la clase magistral sin apoyo de medio audiovisual es aplicada por docentes con edad entre 51 y 60 años y consideran el papel del docente solo como acompañante dentro del proceso de enseñanza aprendizaje. Los docentes que aplican la metodología didáctica de exposición en su mayoría están entre 31 y 40 años y consideran el papel del docente como asesor en el proceso de enseñanza aprendizaje, lo cual justifica su metodología aplicada. Las metodologías didácticas de taller de reconocimiento y clase magistral con apoyo de medio audiovisual se complementan dentro del proceso. Los docentes que las aplican se caracterizan por tener tiempo de vinculación de seis a diez años, nivel de formación
Tabla I. Características de los docentes en 2006

\begin{tabular}{|c|c|c|}
\hline $20-30$ & 15 & (35.7) \\
\hline $31-40$ & 13 & (30.9) \\
\hline $4 I-50$ & 6 & (14.3) \\
\hline $51-60$ & 6 & $(14.3)$ \\
\hline más de 61 & 2 & $(4.8)$ \\
\hline \multicolumn{3}{|c|}{ Vinculación con la FUCS (años) } \\
\hline 1 & 9 & $(21.4)$ \\
\hline $2-5$ & 16 & (38.1) \\
\hline $6-10$ & 8 & (19) \\
\hline $11-15$ & 7 & (16.7) \\
\hline$>16$ & 1 & $(2.4)$ \\
\hline no responde & 1 & $(2.4)$ \\
\hline \multicolumn{3}{|c|}{ Nivel académico } \\
\hline pregrado & 14 & (33.3) \\
\hline postgrado & 27 & $(64.2)$ \\
\hline no responde & 1 & $(2.3)$ \\
\hline
\end{tabular}

Tabla 2. Métodos didácticos utilizados en 2006 Métodos didácticos utilizados con mayor frecuencia

n $\%$

\begin{tabular}{|l|c|c|}
\hline taller de reconocimiento de instrumental & 16 & $(38.1)$ \\
\hline clase magistral con apoyo audiovisual & 18 & $(42.9)$ \\
\hline clase magistral sin apoyo audiovisual & 7 & $(16.7)$ \\
\hline exposición & 9 & $(21.4)$ \\
\hline taller interactivo & 6 & $(14.3)$ \\
\hline no responde & 2 & $(4.7)$ \\
\hline
\end{tabular}

Otros métodos didácticos utilizados

\begin{tabular}{|l|c|c|}
\hline clase magistral & 21 & $(50)$ \\
\hline talleres y maquetas & 17 & $(40.5)$ \\
\hline clase magistral con participación del estudiante & 8 & $(19)$ \\
\hline exposiciones & 7 & $(16.7)$ \\
\hline no responde & 14 & $(33.3)$ \\
\hline
\end{tabular}

\begin{tabular}{|l|c|c|}
\hline \multicolumn{3}{|c|}{ Tabla 3. Papel del docente en 2006} \\
\hline \multicolumn{1}{|c|}{ Papel del docente } & $\mathbf{n}$ & $\%$ \\
\hline planificador & 13 & $(31 \%)$ \\
\hline transmisor de conocimientos & 8 & $(19 \%)$ \\
\hline asesor en el proceso & 20 & $(47.6 \%)$ \\
\hline acompañante & 13 & $(31 \%)$ \\
\hline
\end{tabular}




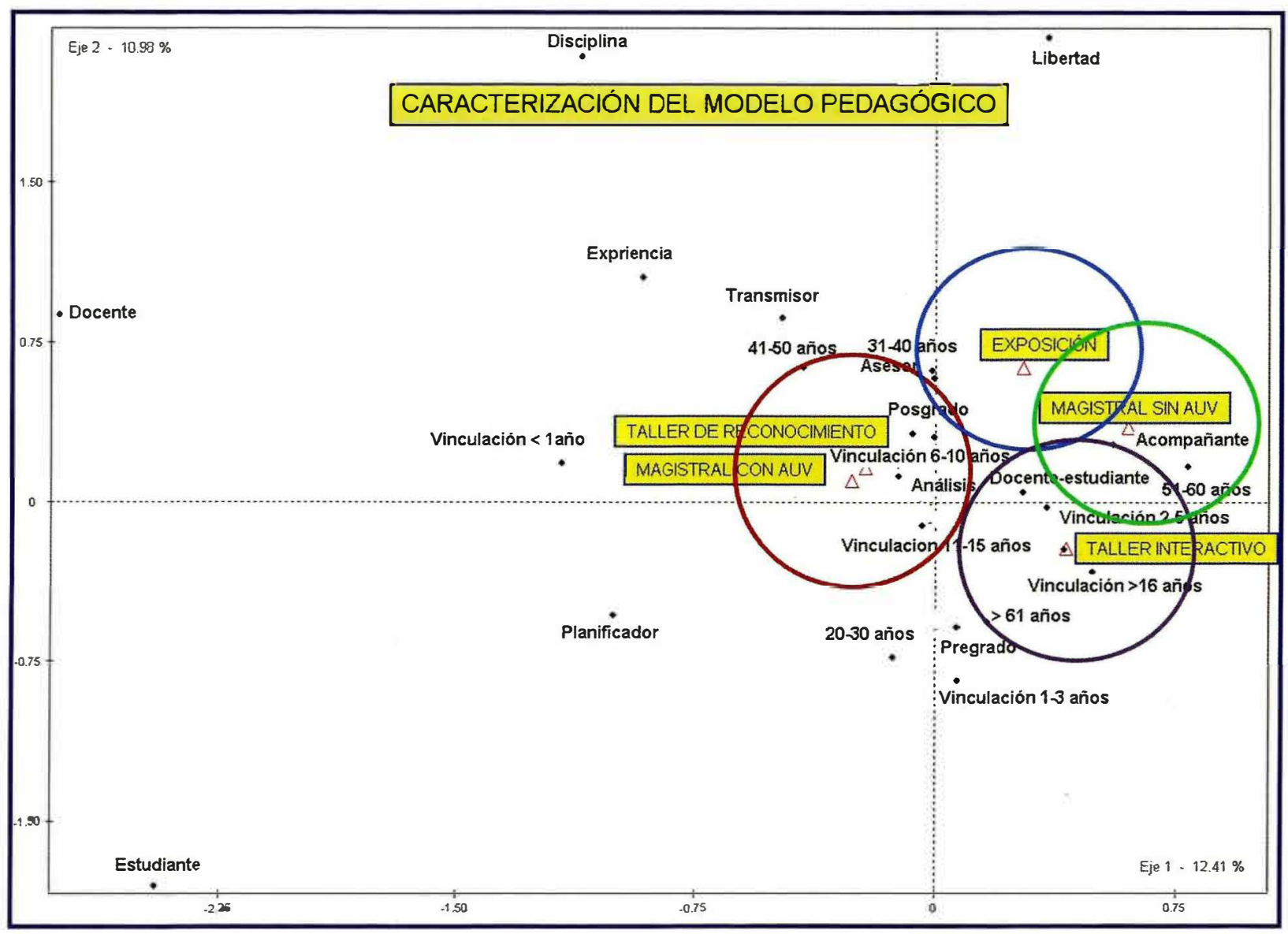

Figura I. Plano factorial. La gráfica describe la relación entre las variables consideradas en el estudio (activas) y los métodos didácticos mas utilizados (ilustrativas). La relación entre las variables se establecen por la cercanía geométrica entre ellas, es decir, entre más cercanas se encuentren mayor será su relación.

académica de posgrado y consideran el papel del docente como transmisor de información dentro del proceso de enseñanza aprendizaje, lo cual justifica la metodología aplicada, ya que el taller de reconocimiento permite solo identificar características de determinado elemento médico quirúrgico.

\section{Discusión}

En cuanto a la metodología didáctica aplicada en instrumentación quirúrgica, es de destacar que en 1997 se realizó un diagnóstico sobre la enseñanza de esta profesión, enfatizando como conclusión que se debe orientar hacia una fusión entre la teoría y la práctica, basada en una reflexión pedagógica permanente di- rigida hacia la construcción del conocimiento. ${ }^{10}$ Sin embargo, para esta época no se evidenció ninguna transformación en cuanto a la orientación pedagógica.

En 2006 se realizó el estudio sobre la caracterización del modelo pedagógico desarrollado por los docentes, el cual mostró que la clase magistral corresponde a la metodología más frecuente utilizada en la facultad, lo cual no favorece el cumplimiento de los objetivos para el estudiante del programa ni corresponde a la metodología propia del aprendizaje significativo establecido en el PEI de la FUCS. Por lo tanto, es importante que los docentes consideren otro tipo de metodología que favorezca el proceso de enseñanza aprendizaje de los estudiantes del programa. 
A partir de estos resultados, los docentes de las asignaturas de fundamentación específica de la facultad de instrumentación quirúrgica fortalecen su metodología didáctica con la aplicación de la simulación creativa como didáctica específica para la enseñanza de esta profesión. En este tipo de simulación el estudiante construye los órganos del cuerpo humano en diferentes materiales para simular sobre ellos los pasos de una técnica quirúrgica, con instrumental y elementos médico quirúrgicos reales. Se desarrolla en tres fases, la observación, reflexión, y planeación y ejecución, de esta manera se propicia un ambiente para el análisis, la integración de nuevos conceptos a las estructuras cognitivas previas y la construcción de un nuevo conocimiento, favoreciendo la intencionalidad del programa y respondiendo a los requerimientos de una profesión que se encuentra en auge. Es una contribución tanto para la FUCS como para las demás facultades de instrumentación quirúrgica en Colombia.

En cuanto a los demás criterios que caracterizan un modelo pedagógico como son el papel del docente, las orientaciones dentro del proceso de enseñanza aprendizaje y las estrategias de construcción microcurricular, es importante resaltar que según el estudio realizado estos criterios se encuentran de acuerdo con las características de un modelo pedagógico constructivista y el aprendizaje significativo establecido en el PEI de la FUCS.

\section{Referencias}

1. Colombia. Congreso de la República. Ley 784 de 2002, por medio de la cual se reforma la Ley 6a. del 14 de enero de 1982. Diario Oficial No 45.046, (Diciembre 27 de 2002)

2. Colombia. Congreso de la República. Ley 115 de 1994, Por la cual se expide la ley general de educación. Diario Oficial No. 41.214, (Febrero 8 de 1994)

3. Colombia. Ministerio de Educación Nacional. Creación de programas académicos[monografía en Internet]. Bogotá: El Ministerio: 2010. [citado22 Mar. 2013]. Disponible en: http://www.mineducacion.gov.co/l621/w3-article-235796.html

4. Colombia. Congreso de la República. Ley 1188 de 2008 Por la cual se regula e registro calificado de programas de educación superior y se di tan otras disposiciones. Diario Oficial. 46971 (abril 252()$(08)$.

5. Ontoria A. Aprendizaje centrado en el alumno: metodología para una escuela abierta. Madrid, España: Narcea; 2006.

6. Fundación Universitaria de Ciencias de la Salud. Proyecto educativo institucional. [monografía en Internet]. Bogotá: FUCS; 2008. [citado22 Mar. 2013]. Disponible en: http://www.fucsalud.edu.co/pdf/documentosimportantes/PEI_FUCS.pdf

7. Colombia. Ministerio de Educación Nacional. Resolucion 2772. Por la cual se definen las características específicas de calidad para los programas de pregrado en ciencias de la salud. Diario Oficial No. 45.388, (diciembre 1 de 2003)

8. Flórez R. Hacia una pedagogía del conocimiento.2a ed. Madrid: MacGrawHill;2005

9. De Zubiría J. Tratado de pedagogía conceptual: los modelos pedagógicos. Bogotá: fundación Internacional de Pedagogía Conceptual Alberto Merani; 2012.

10. Carreño Chamorro D, Cortés Cárdenas S, Páez Castro N, Rey Hurtado B, Romero Rozo D. Hacia una didáctica en la instrumentación quirúrgica [tesis]. Bogotá Fundación Universitaria de Ciencias de la Salud. Facultad de Instrumentación Quirúrgica; 1997 